Exploiting the optical reflectance behaviour of carbon fibre composites for low-cost inspection and orientations analysis

Pierce, R.S., Liu, X.

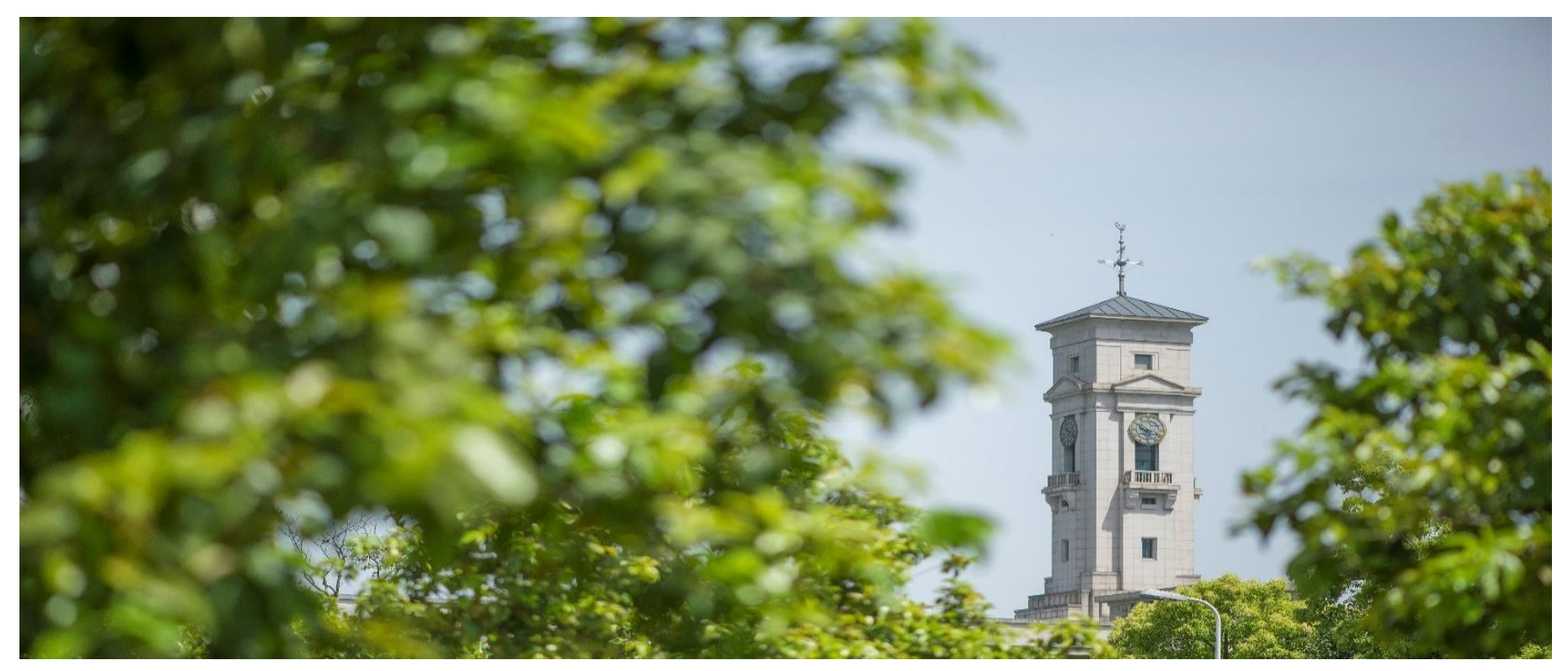


University of Nottingham Ningbo China, 199 Taikang East Road, Ningbo, 315100, China

First published 2020

This work is made available under the terms of the Creative Commons Attribution 4.0 International License:

http://creativecommons.org/licenses/by/4.0

The work is licenced to the University of Nottingham Ningbo China under the Global University Publication Licence:

https://www.nottingham.edu.cn/en/library/documents/researchsupport/global-university-publications-licence.pdf Nottingham 


\title{
Exploiting the optical reflectance behaviour of carbon fibre composites for low-cost inspection and orientations analysis
}

\author{
Robert S. Pierce ${ }^{\mathrm{a}^{*}}$ and Xiaoling Liu ${ }^{\mathrm{a}}$
}

${ }^{a}$ Department of Mechanical, Materials and Manufacturing Engineering, University of Nottingham Ningbo China, University Park, Ningbo, P.R. China, 315100.

* Corresponding author. Email: Robert.pierce@nottingham.edu.cn

\begin{abstract}
This work focusses on understanding and exploiting the optical reflectance behaviour of carbon fibre reinforced polymer (CFRP) composites for the non-destructive determination of fibre orientation and surface defects. By taking a series of images under different lighting conditions, fibre reflections have been isolated and MATLAB image analysis has been used to calculate the fibre orientations across the surface of the composite. An Average Peak Method has been developed and validated against manual orientation measurements for two different applications: a machined scarf repair surface and a discontinuous fibre composite. The same experimental method and analysis code showed good results for both cases without modification, when at least 16 images with different directional lighting conditions are used. Hence, this lowcost approach can be applied to a diverse range of quality assurance and research applications where fibre orientations or other surface details need to be characterised.
\end{abstract}

Keywords: Inspection, Repair, Quality Assurance, SMC.

\section{Introduction}

Reliable inspection techniques can be used to detect flaws, monitor quality, and trace parts through composites production lines and in research laboratories. Due to the anisotropic behaviour of fibre reinforced composites, it can be particularly important to understand local fibre orientations as they can significantly affect part performance. 
A range of inspection technologies and processing techniques have been previously studied for the characterisation of fibre orientations in composite materials, although microscopic [1] and radiographic (X-ray) imaging methods [2] are conventionally used. Microscopic methods commonly rely on small samples to be sectioned perpendicular to the approximate fibre direction, from which any elliptical deviation from the ideal circular fibre shape is assumed to correlate with the fibre orientation. A study by Kratmann et al. [3] demonstrated that alternative image analysis methods (Fourier transform misalignment analysis or multiple field image analysis) of micrographs parallel to the fibre direction can achieve $\pm 0.2^{\circ}$ resolution of the mean fibre orientation, similar to common elliptical analysis methods. However, all such methods are generally destructive and require some advance knowledge of the fibre orientations for the most accurate analysis.

Common Non-Destructive Inspection (NDI) methods, such as ultrasound, are generally unable to determine fibre orientations due to resolution limitations. Although some effort has been made to characterise out-of-plane wrinkling inside continuous fibre reinforced composites with focussed probes $[4,5]$ and laser ultrasound methods [6]. Some success for in-plane orientation measurement has also been reported using active thermography and ultrasonic spectroscopy with guided Lamb waves [7], but was specific to a glass fibre Sheet Moulded Compound (SMC) material reinforced by carbon fibre tapes. Other research has considered radio frequency eddy current technologies and 2D Fast Fourier Transforms to non-destructively analyse the texture and orientation of woven carbon fibre reinforced polymers for quality assurance purposes [8].

Radiographic methods typically rely on Micro Computed Tomography (CT) for the necessary resolution to identify individual fibre bundles and to produce a 3D representation of the material for subsequent fibre orientation analysis. Although it is common to reduce the 3D representation to 2D slices to facilitate Gaussian filtering methods. Such an approach has been evaluated against an ultrasonic Scanning Acoustic Microscopy (SAM) method to assess fibre orientations through the thickness of an SMC composite [9]. The two approaches showed reasonable agreement and 
relatively consistent results (around $\pm 5^{\circ}$ ) for the principal direction of anisotropy through the thickness of the sample. In other work, comparing optical microscopy and radiographic methods for determining fibre orientations in short fibre reinforced polymers, Bernasconi et al. [10] have shown that both can produce accurate and similar results under ideal conditions. Generally, due to scale limitations in Micro-CT devices, radiographic samples are often sectioned from larger specimens.

Furthermore, like most other methods, radiographic approaches also rely on representative sampling to produce a probabilistic determination of fibre orientations in larger samples.

Alternatively, simple camera systems and image filtering techniques have been used to determine fibre misalignment across the surface of large unidirectional prepreg plies, prior to composite layup and consolidation [11]. This work relied on the edge detection of individual fibres or bundles at high resolution in smaller subset areas to calculate the local mean fibre orientation. A similar method taking images of subset areas $\left(75 \times 75 \mathrm{~mm}^{2}\right)$ under diffuse lighting conditions has been used to evaluate the fibre distribution in glass fibre SMCs [12]. In application, commercial laser and imaging systems for automated quality control also exist, such as the Hexagon Manufacturing Intelligence Composite Inspection System, however these are expensive and closed systems that require specialised software to operate.

In other fields, optical reflectance behaviour has been studied for computer graphics [13] and reflector materials [14]. Laser- [15] and image-based [16] measurement systems have both been used to measure light distributions from hemispherical directions in order to generate the full reflectance profile of a material. However, these systems tend to be slow and assume a uniform behaviour for the material based on measurements taken from a small region of interest.

Ultimately, very little research has considered image analysis of composites by taking advantage of their optical reflectance behaviour. Feraboli et al. [17] demonstrate one such approach with images of a discontinuous carbon fibre composite coupon taken at different angles to estimate the random nature of the chip distribution. However, a more thorough investigation of composite reflectance is warranted. This work aims to 
understand and exploit this behaviour, and to demonstrate the potential for rapid and low-cost inspection with a focus on determining local fibre orientations across large areas.

\section{Theory}

The anisotropic structure of Carbon Fibre Reinforced Polymer (CFRP) materials that makes them useful for tailoring mechanical behaviour, also results in unique reflectance properties. At the microscale, reinforcement fibres appear as relatively smooth, high aspect-ratio cylinders embedded within a translucent polymer matrix. Figure 1 shows an optical micrograph of the machined surface of a CFRP laminate at the interface between two perpendicular plies. The long and aligned cylindrical fibres of each ply can be clearly observed, even beneath the polymer matrix.

A close examination of the CFRP microstructure reveals some interesting reflectance behaviour that can be summarised by a few key observations in Figure 2:

A. Firstly, when the direction of the light source is perpendicular to the fibre orientation, the changing normals $\left(\mathrm{N}_{1, \mathrm{f}}\right)$ around the circumference of the fibres allow for reflections at many different angles (demonstrated by $R_{1, f}$ ). An observer in the same plane as the fibre surface normal and the light source can therefore receive simultaneous reflections from many parallel fibres that are oriented perpendicular to this plane.

B. Under the same lighting conditions the composite surface, with its constant normal, $\mathrm{N}_{\mathrm{c}}$, will produce a constant reflection, $\mathrm{R}_{1, \mathrm{c}}$.

C. Any other position of the light source relative to the fibre orientation will not produce fibre reflectance behaviour that is significantly different to the composite surface reflectance. This is illustrated by the extreme case where the direction of the light source, surface normals (of both fibre and composite), and fibre orientation are all co-planar. Here the reflectance behaviour of the fibre surface is coincident with that of the composite $\left(R_{2, f}=R_{2, c}\right)$ For an observer, this means the individual fibre reflectance will not be distinguishable from the reflectance behaviour of the composite surface. 
This reflectance behaviour can therefore be exploited to isolate fibre reflections without interference from the composite surface reflections by considering two main factors:

1. The position of the light source, observer and point of interest should form a plane that is perpendicular to the fibre orientation.

2. The observer should be positioned in order to avoid as much of the composite surface reflectance as possible while still being able to observe a strong reflection from the fibres.

\section{Application}

Based on the above understanding of CFRP reflectance behaviour, a robust method has been developed to characterise fibre orientations at every point on a composite surface. This approach relies on a stationary camera that is positioned facing normal to the stationary composite sample. A series of images are taken of the composite surface subject to light from different orientations (regularly spaced from $0^{\circ}$ to $360^{\circ}$ at a constant radius). A shallow angle of incidence for the light source relative to the area of interest helps to eliminate composite surface reflections and isolate only the fibre surface reflections. Hence, the quality of the reflections and images will be dependent upon the radius of the area of interest, $r$, the distance of the camera from the area of interest, $c$, and the position of the light source (distance from area of interest, $d$, and height from surface, $h$ ). Figure 3 illustrates this relationship between the camera position, light source and area of interest. If $\gamma=0^{\circ}$, then $\alpha=\beta$ and a strong reflection will be observed from the composite surface at the edge of the area of interest, obscuring any fibre reflections. In order to avoid this, a minimum tolerance value of $\gamma=20^{\circ}$ is recommended. This value has been selected based on observations from samples with relatively smooth surfaces (as are seen in the examples of Section 4). Rougher surfaces may result in a more diffuse reflection for which a larger tolerance value of $\gamma$ may become necessary. Also, if $\alpha$ (the angle between the light source and composite surface normal) is too large, there may be significant occlusion of the light from adjacent fibres, and fibre reflections may have a reduced intensity. 
In practice, it is likely that the area of interest and camera height may be known, but a suitable position of the light source needs to be determined. To this end, the following geometric relationship can be used to find a suitable light source location:

$$
\frac{d}{h} \geq \frac{\frac{r}{c}+\tan \gamma}{1-\frac{r}{c} \tan \gamma}
$$

The size of the light source is also recommended to be larger than the area of interest for the best results, however this too will introduce undesirable composite surface reflections if the distance of the light source from the area of interest is relatively small.

\subsection{Image analysis code}

The images taken under different lighting conditions can then be analysed. In this case MATLAB has been used and each pixel is considered as a discrete point. Since all images are taken with fixed sample and camera positions, the greyscale intensity value, $P_{i}^{k}$ (between 0 and 255), for each pixel, $i$, from each image, $k$, will always correspond with the same point on the sample surface. Two different methods have been developed to determine the fibre orientation at each point.

The first approach, termed the Maximum Peak Method, is the simplest interpretation of the above theory. By observing the lighting orientation, $\theta^{k}$, (from the corresponding image, $k$ ) that produces the greatest reflection intensity at each point, the fibre orientation is predicted by the Maximum Peak Method, $\varphi_{i}^{\operatorname{Max}}$, according to Eq. (2). This assumes that the fibre direction is perpendicular to the plane of the light and observer the produces the maximum reflection intensity. Here $M_{i}$ is an integer value (between 1 and $n$ ) that identifies the image with the maximum greyscale intensity for the $i^{\text {th }}$ pixel, and $n$ is the total number of images in the series (recommended to be a factor of 4 , and a minimum of 8 images).

$$
\varphi_{i}^{M a x}=\theta^{M_{i}}+90^{\circ}=\frac{M_{i}-1}{n} \times 360^{\circ}+90^{\circ}
$$


The second approach, termed the Average Peak Method, has been developed to make use of more data and to produce a more accurate measurement of the fibre orientation at any point. Fundamentally, this is an extension of the Maximum Peak Method that searches for the image with a second local maxima of pixel intensity, $m_{i}$, from a subset range of images, $r_{i}$, with light orientations diametrically opposed to that which produces the maximum peak, $M_{i}$. The image search range is defined by Eq. (3).

$$
1+\left(M_{i}+\frac{n}{4}-1\right) \bmod n \leq r_{i} \leq 1+\left(M_{i}+\frac{3 n}{4}-1\right) \bmod n
$$

Once the two local maxima, $M_{i}$ and $m_{i}$, have been identified for any given pixel, then the fibre orientation predicted by the Average Peak Method, $\varphi_{i}^{A v g}$, is assumed to be perpendicular to the average of these two opposite peaks according to Eq. (4).

$$
\varphi_{i}^{A v g}=\left[\left(\frac{M_{i}+m_{i}-2}{n}+0.5\right) \bmod 1\right] \times 180^{\circ}+90^{\circ}
$$

A flow chart for these operations is shown in Figure 4. Figure 5 depicts a practical example of the Average Peak Method at a single point. Here the maximum reflection intensity for a given pixel is observed with a light orientation of $15^{\circ}$, and a second maxima is observed at $235^{\circ}$. The Maximum Peak Method would predict the fibre orientation to be $105^{\circ}$ based on the single $15^{\circ}$ peak, however the Average Peak Method instead measures the fibre orientation based on the mean direction of two opposing maxima, which would be $125^{\circ}$ (or $305^{\circ}$ ).

After the predicted fibre orientations from each method are identified and stored, the results are best visualised using a periodic HSV (Hue Saturation Value) colour model. This cycles through red-yellow-green-cyan-blue-magenta-red to allow for a distinct RGB colour value to be attributed to any fibre orientation between $0^{\circ}$ and $180^{\circ}$.

\section{Example cases}


In order to demonstrate these methods, two different CFRP applications have been investigated. The first case looks at machined scarf surface of a laminated continuous reinforced composite, representative of an aircraft repair. The second application considers the surface of a Sheet Moulding Compound (SMC) made from discontinuous carbon fibres and epoxy resin, representative of a material that might be used for automotive parts. The versatility of this automatic inspection technique is demonstrated by using the same experimental configuration and MATLAB code for both applications without modification. Here both cases are limited to surface measurements from relatively flat carbon fibre reinforced epoxy composites. However, the same approach may be suitable for other fibre reinforced composite materials and the theoretical understanding could easily be extended to a scanning 3D robotic system that focuses only on a small region of interest at a time and compiles a stitched 3D representation of the surface fibre orientations.

\subsection{Experimental configuration}

Inspection experiments were all carried out in a dark room using a 285x190 mm LED monitor with a luminous intensity of 374 nits as the single light source. Composite samples were placed on the middle of a horizontal surface with a 16-megapixel camera (28.26 $\mathrm{mm}$ full-frame equivalent focal length) fixed in place $500 \mathrm{~mm}$ directly above the observation surface. The camera settings were kept consistent for each series of images and selected for greatest contrast without over/under saturation (ie. aperture f/2.0, 0.5 sec shutter time, ISO-250). The light source (LED screen) was positioned vertically, at a distance of $600 \mathrm{~mm}$ from the centre of the sample, with the lower edge elevated $25 \mathrm{~mm}$ from the horizontal surface. In order to highlight the fibre reflections from 32 different light orientations, the light source was rotated in increments of $11.25^{\circ}(\pi / 16 \mathrm{rad})$ about the centre of the sample at a constant distance. Images were taken under each different condition to make up a full series of 32 input images for the MATLAB image analysis outlined in Section 3.1.

\subsection{Machined scarf repair surface}


The first application is related to the repair of aerospace composite parts, where bonded scarf repairs remain a considerable certification challenge [18]. This is also an area for active research in automated machining to produce optimised bonding geometries [19]. Such machining could benefit from a fast and reliable technique for determining ply orientations, deviations or defects, across the scarf area, regardless of whether the work is done by hand in the field or by robotic tools in a depot.

A $225 \times 255 \mathrm{~mm}$ demonstrator panel was prepared from a representative wing skin laminate and machined with an optimised scarf shape [20] for the inspection experiment. The complex scarf was machined with a nominal taper of $3^{\circ}$, around a simulated damage area of $80 \mathrm{~mm}$ diameter, and to a depth of one-third of the total panel thickness. After a solvent clean, a series of 32 images were taken with the varying light source orientations. A subset of eight images can be seen in Figure 6, highlighting the strong reflections that are observed from the surface of fibres (perpendicular to the plane of the light source and camera). The images have been automatically processed using MATLAB to measure the fibre orientation at any point across the surface of the demonstrator panel. Figure 7 shows the results determined from both the Maximum Peak Method (left) the Average Peak Method (right) against the same HSV colour map. Both methods produce similar results in the middle of the panel, however the Maximum Peak Method results further from the centre are significantly influenced by the changing light position. By contrast, the Average Peak Method results appear more reliable and unaffected by the change in light orientation.

A histogram of the Average Peak Method results is shown in Figure 8, highlighting the dominance of $90^{\circ}$ fibres across the surface along with significant peaks at $0^{\circ}\left(180^{\circ}\right), 45^{\circ}$ and $135^{\circ}$. For materials with an unknown stacking sequence, this approach could quickly identify the ply orientations and ply boundaries to facilitate the optimal design of the repair patch.

Aside from the measurement of fibre orientations across the sample, this inspection approach also allows for the identification of anomalies on the sample surface. Closer inspection of one corner of the Average Peak Method results, shown in Figure 9, 
reveals a number of interesting details. Surface scratches, embedded offcut fibre trimmings, areas of spilled resin contamination and the edge of the machining area can all be identified.

\subsection{Discontinuous fibre composite surface}

The second example case focuses on discontinuous fibre composites that might be used in the production of automotive parts. In this field, the location and alignment of fibre bundles within a composite can have a significant effect on the overall performance and failure [21]. Hence it can be useful to understand the orientation of fibre bundles both from individual preform layers prior to consolidation and from the final composite surface.

In this case, a square SMC panel $(410 \times 410 \mathrm{~mm})$ has been photographed 32 times under different lighting conditions for subsequent image analysis. Eight images from various lighting orientations are shown in Figure 10. Compared with the machined repair surface case, the proximity of the light source here has a significant influence on the surface reflection behaviour at the edges of the sample.

The fibre orientation results, from image analysis using both the Maximum (left) and Average (right) Peak Methods, are shown in Figure 11. Again, the two methods produce similar results in the centre of the sample, and the outer values for the Maximum Peak Method are greatly influenced by the changing light position while the Average Peak Method results seem much more realistic. A histogram of the Average Peak Methods results is shown in Figure 12, suggesting a relatively random distribution of the fibres in this case, with a slight bias between $90^{\circ}$ and $135^{\circ}$. This type of rapid image analysis is expected to be particularly useful for research investigating fibre alignment in discontinuous fibre-reinforced composites [22].

\section{Validation}

In order to evaluate the accuracy of these fibre orientation predictions, manual fibre orientation measurements were also taken (where possible) from a regular $10 \times 10$ grid of points across the surfaces of both samples, as indicated in Figure 13. At several 
the regular grid points, the fibre orientation could not be measured manually as these points coincided with gaps between tows or resin rich areas where there was no clear fibre direction. A total of 88 and 71 points from the $10 \times 10$ grid were 'measurable' for the machined scarf and discontinuous fibre composite samples respectively. To assess the variability of the manual measurement process itself, $2^{\text {nd }}$ and $3^{\text {rd }}$ repeat measurements were taken at measurable points. The mean standard deviation for manual measurements at any given point was found to be $1.51 \%$.

Figure 13 also depicts an inverse representation of reflection intensity that is useful for identifying areas of lower peak intensity. These indicate areas where the calculated fibre orientations may not be as accurate, and often correspond physically with resin rich areas where the fibre reflection is dulled by a translucent layer of resin (such as the unmachined surface of the repair panel).

The results of the Maximum and Average Peak Methods have been compared against manual measurements in Figure 14 and Figure 15 for the machined scarf and discontinuous fibre composite surfaces respectively. In both cases the Average Peak Method shows better clustering and agreement with the manual measurements than the Maximum Peak Method.

The mean variance of the calculated fibre orientations from measured values, for both methods and both applications, has been evaluated using 8, 16 and 32 images, as shown in Figure 16. Generally, the mean variance is seen to reduce with a greater number of images, although beyond 16 images there may not be a significant benefit. The Average Peak Method is also seen to have half the mean variance of the Maximum Peak Method, except in the case of 8 images for the machined scarf repair. This is due to the coincidental alignment of the 8 light orientations with the actual fibre directions, as seen in Figure 6. The variance for the Maximum Peak Method is therefore expected to be greater when the lighting positions are not aligned with the four main fibre orientations.

\section{Conclusions}


A theoretical basis for rapid and low-cost scanning of composite surfaces has been developed by exploiting the unique optical reflectance properties of carbon fibre reinforced polymer composites. Fundamentally, fibre reflections can be isolated from surface reflections under particular conditions where the fibre direction is perpendicular to the plane made by the light source, point of interest and observer. Based on this understanding, an application for determining surface fibre orientations has been introduced by taking a series of images taken under different lighting conditions with a stationary sample and camera position. Two different image analysis methods have been developed for a universal MATLAB code that automatically interprets the image series. Through the study of two different experimental cases, the Average Peak Method has proven to be more reliable than the simpler Maximum Peak Method when compared to manual measurements of fibre orientations.

Recommendations on camera, sample and lighting positions for improved results have been outlined and a minimum of 16 images, taken under regularly spaced lighting orientations (every $22.5^{\circ}$ ), is advised. The developed methods can also be used to reveal surface anomalies.

\section{Acknowledgements}

This work was done in the "ACC TECH-UNNC joint laboratory in Sustainable Composite Materials". The authors would like to acknowledge the financial support from Ningbo S\&T bureau under Collaboration Project (Project code: 2017D10033), Ningbo 3315 Innovation team Scheme and Zhejiang Innovation Team scheme. The authors would also like to acknowledge the initial investigation and work by Ahmad A. E. Tello that helped seed some of the fundamental aspects of this research.

\section{References}

[1] S. W. Yurgartis, Measurement of small angle fiber misalignments in continuous fiber composites, Compos. Sci. Technol., vol. 30, no. 4, pp. 279-293, (1987).

[2] E. G. Kim, J. K. Park, and S. H. Jo, A study on fiber orientation during the injection molding of fiber-reinforced polymeric composites: (Comparison between image 
processing results and numerical simulation), J. Mater. Process. Technol., vol. 111, no. 1-3, pp. 225-232, (2001).

[3] K. K. Kratmann, M. P. F. Sutcliffe, L. T. Lilleheden, R. Pyrz, and O. T. Thomsen, A novel image analysis procedure for measuring fibre misalignment in unidirectional fibre composites, Compos. Sci. Technol., vol. 69, no. 2, pp. 228238, (2009).

[4] L. J. Nelson, R. A. Smith, and M. Mienczakowski, Ply-orientation measurements in composites using structure-tensor analysis of volumetric ultrasonic data, Compos. Part A Appl. Sci. Manuf., vol. 104, pp. 108-119, (2018).

[5] L. J. Nelson and R. A. Smith, Fibre direction and stacking sequence measurement in carbon fibre composites using Radon transforms of ultrasonic data, Compos. Part A Appl. Sci. Manuf., vol. 118, no. April 2018, pp. 1-8, (2019).

[6] L. Ambrozinski, J. Mrowka, M. O’Donnell, and I. Pelivanov, Detection and imaging of local ply angle in carbon fiber reinforced plastics using laser ultrasound and tilt filter processing, Compos. Part A Appl. Sci. Manuf., vol. 126, no. August, p. 105581, (2019).

[7] M. Zaiß, M. H. Jank, U. Netzelmann, T. Waschkies, U. Rabe, H. G. Herrmann, M. Thompson, and G. Lanza, Use of Thermography and Ultrasound for the Quality Control of SMC Lightweight Material Reinforced by Carbon Fiber Tapes, in Procedia CIRP, (2017), vol. 62, pp. 33-38.

[8] H. Heuer, M. Schulze, M. Pooch, S. Gäbler, A. Nocke, G. Bardl, C. Cherif, M. Klein, R. Kupke, R. Vetter, F. Lenz, M. Kliem, C. Bülow, J. Goyvaerts, T. Mayer, and S. Petrenz, Review on quality assurance along the CFRP value chain - Nondestructive testing of fabrics, preforms and CFRP by HF radio wave techniques, Compos. Part B Eng., vol. 77, pp. 494-501, (2015).

[9] K. Schladitz, A. Büter, M. Godehardt, O. Wirjadi, J. Fleckenstein, T. Gerster, U. Hassler, K. Jaschek, M. Maisl, U. Maisl, S. Mohr, U. Netzelmann, T. Potyra, and M. O. Steinhauser, Non-destructive characterization of fiber orientation in reinforced SMC as input for simulation based design, Compos. Struct., vol. 160, pp. 195-203, (2017). 
[10] A. Bernasconi, F. Cosmi, and P. J. Hine, Analysis of fibre orientation distribution in short fibre reinforced polymers: A comparison between optical and tomographic methods, Compos. Sci. Technol., vol. 72, no. 16, pp. 2002-2008, (2012).

[11] Y. Davila, L. Crouzeix, B. Douchin, F. Collombet, and Y. H. Grunevald, Identification and modelling of the in-plane reinforcement orientation variations in a cfrp laminate produced by manual lay-up, Appl. Compos. Mater., vol. 25, no. 3, pp. 647-660, (2017).

[12] A. Kraemer, S. Lin, D. Brabandt, T. Böhlke, and G. Lanza, Quality control in the production process of SMC lightweight material, in Procedia CIRP, (2014), vol. 17, pp. $772-777$.

[13] H. Li and K. E. Torrance, A Practical, Comprehensive Light Reflection Model, Citeseer, pp. 1-12, (2005).

[14] P. M. Adams, D. R. Alaan, and C. J. Panetta, Debris Characterization, Albedo, and Plume Measurements from Laser Ablations of Satellite Materials in HighVacuum and in Gaseous Ambients, in Advanced Maui Optical and Space Surveillance Technologies Conference, (2017).

[15] M. Janecek and W. W. Moses, Optical reflectance measurements for commonly used reflectors, IEEE Trans. Nucl. Sci., vol. 55, no. 4, pp. 2432-2437, (2008).

[16] S. R. Marschner, S. H. Westin, E. P. F. Lafortune, and K. E. Torrance, Image-based bidirectional reflectance distribution function measurement, Appl. Opt., vol. 39, no. 16, p. $2592,(2000)$.

[17] P. Feraboli, E. Peitso, F. Deleo, T. Cleveland, and P. B. Stickler, Characterization of prepreg-based discontinuous carbon fiber/epoxy systems, J. Reinf. Plast. Compos., vol. 28, no. 10, pp. 1191-1214, (2009).

[18] A. A. Baker, A. J. Gunnion, and J. Wang, On the Certification of Bonded Repairs to Primary Composite Aircraft Components, J. Adhes., vol. 91, no. 1-2, pp. 4-38, (2015).

[19] M. Niedernhuber, J. Holtmannspötter, and I. Ehrlich, Fiber-oriented repair geometries for composite materials, Composites Part B: Engineering. (2016). 
[20] R. S. Pierce and B. G. Falzon, Modelling the size and strength benefits of optimised step/scarf joints and repairs in composite structures, Compos. Part $B$ Eng., vol. 173, p. 107020, (2019).

[21] M. Such, C. Ward, and K. Potter, Aligned Discontinuous Fibre Composites: A Short History, J. Multifunct. Compos., vol. 2, no. 3, pp. 155-168, (2014).

[22] L. T. Harper, T. A. Turner, J. R. B. Martin, and N. A. Warrior, Fiber alignment in directed carbon fiber preforms - Mechanical property prediction, J. Compos. Mater., vol. 44, no. 8, pp. 931-951, (2010). 


\section{List of Figures}

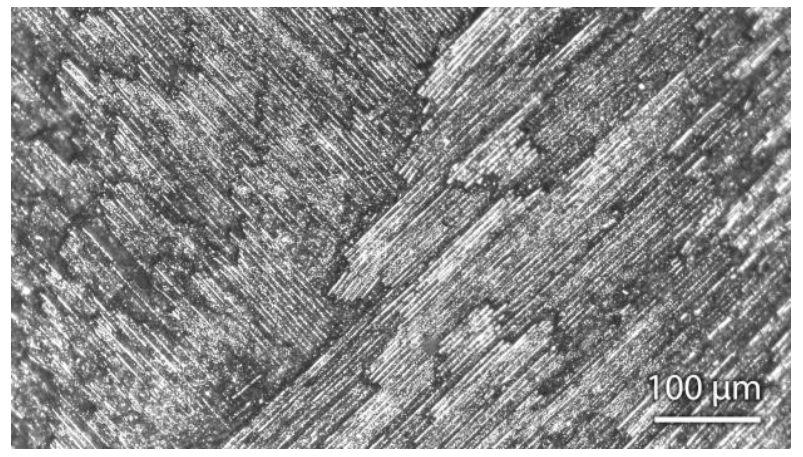

Figure 1: Optical micrograph of reinforcement fibres at the interface of two perpendicular plies from a machined CFRP surface.

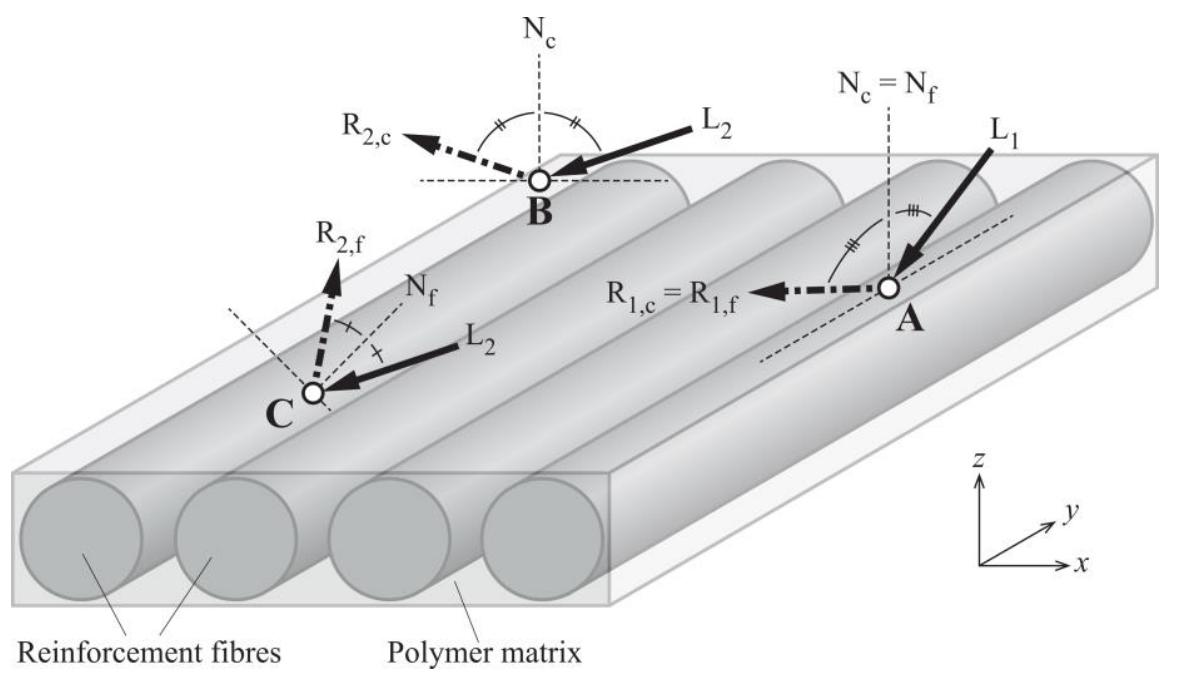

Figure 2: Representation of light reflections from CFRP materials. Where, A), fibre reflection behaviour is distinct from, B), composite surface reflection when the light source is perpendicular to the fibre orientation. However, when the light direction is not perpendicular to fibre orientation, C), reflections may not be distinguishable between fibres and the composite surface.

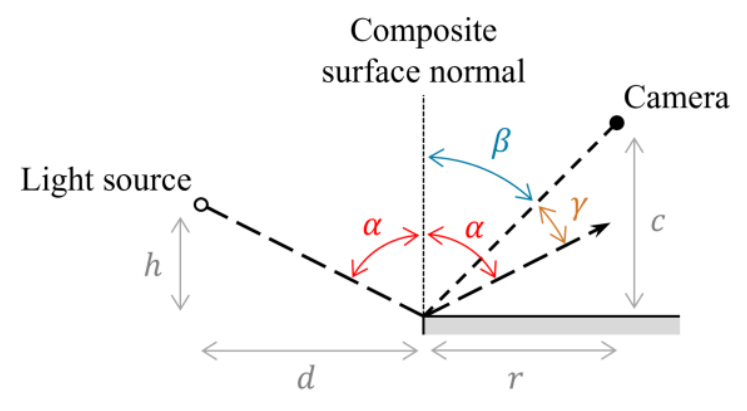

Figure 3: Application geometry for composite surface reflection. 




Figure 4: Flow chart for determination of fibre orientation based on the Maximum Peak and Average Peak Methods.

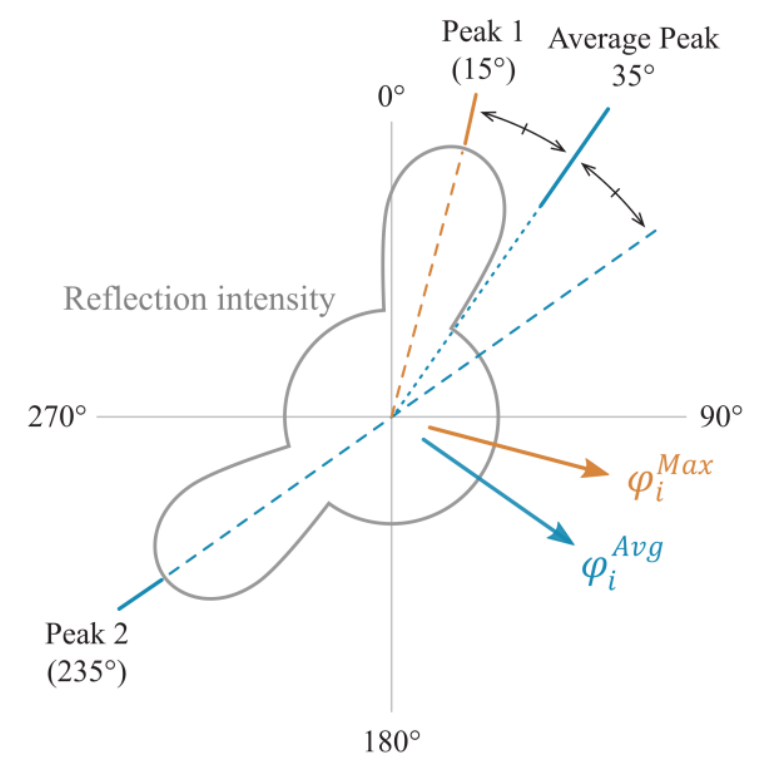

Figure 5: Example of the Average Peak Method for a radial plot of reflection intensity at a single point with respect to different lighting orientations. 

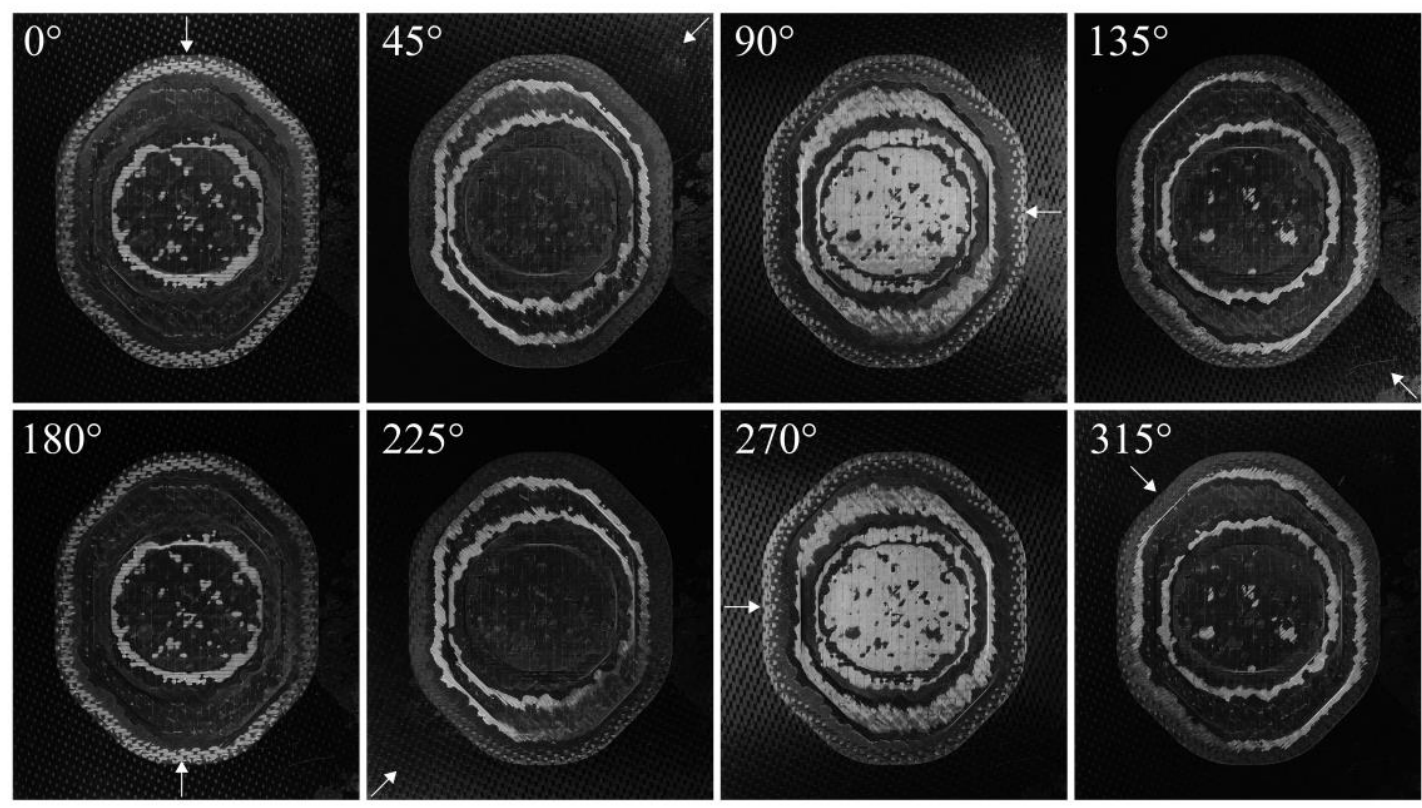

Figure 6: Raw images ( 8 of 32 total) taken with light source positioned at various orientations relative to the machined scarf repair sample (light direction indicated by arrows).

a)

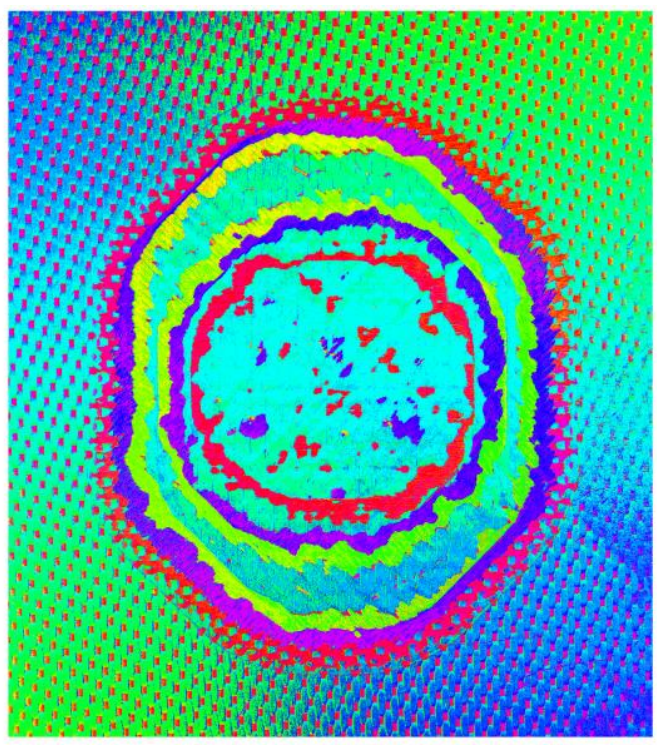

b)

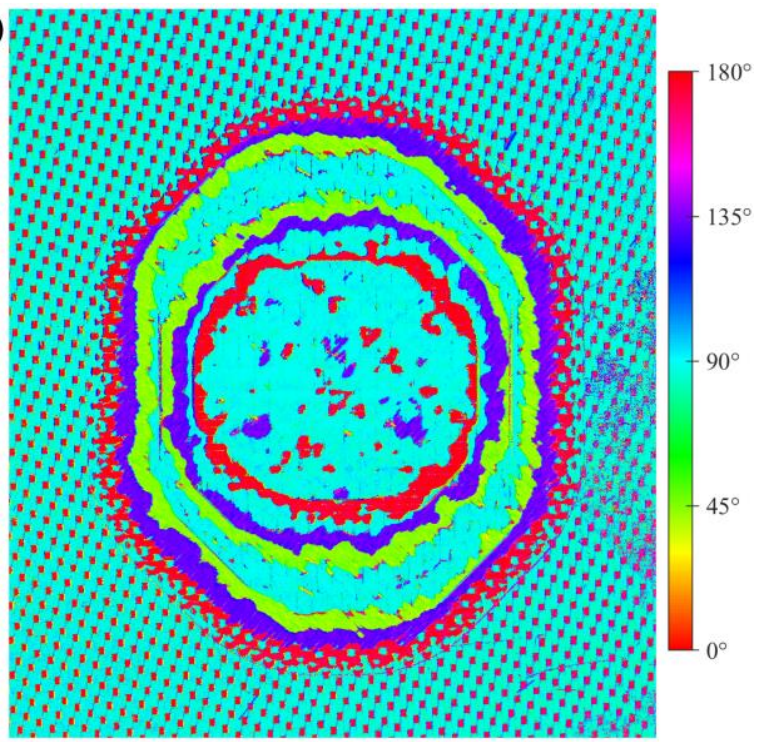

Figure 7: Fibre orientations in the machined scarf repair surface determined by, a) the Maximum Peak Method and, b) the Average Peak Method. 


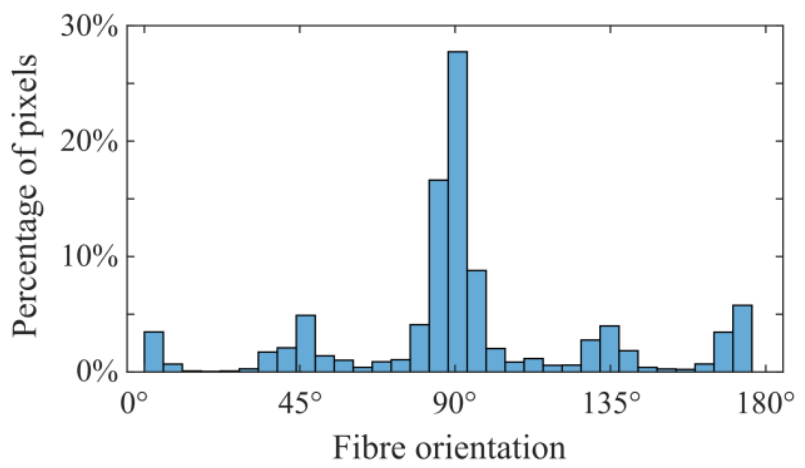

Figure 8: Histogram of fibre orientations across the machined scarf repair surface, determined by the Average Peak Method.

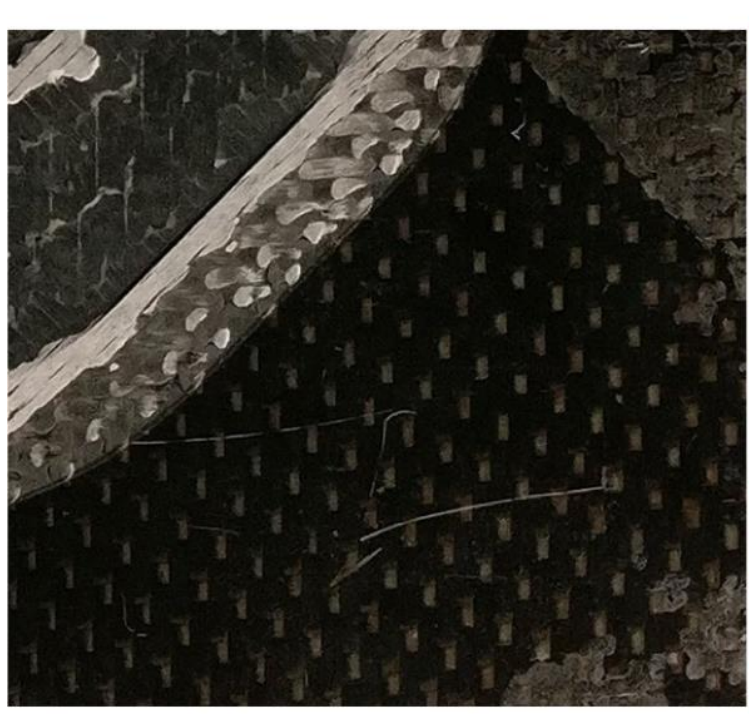

Scratches Resin spill/contamination

Edge of machining Loose offcut fibres

Figure 9: Detail of features seen in the Average Peak Method results. 

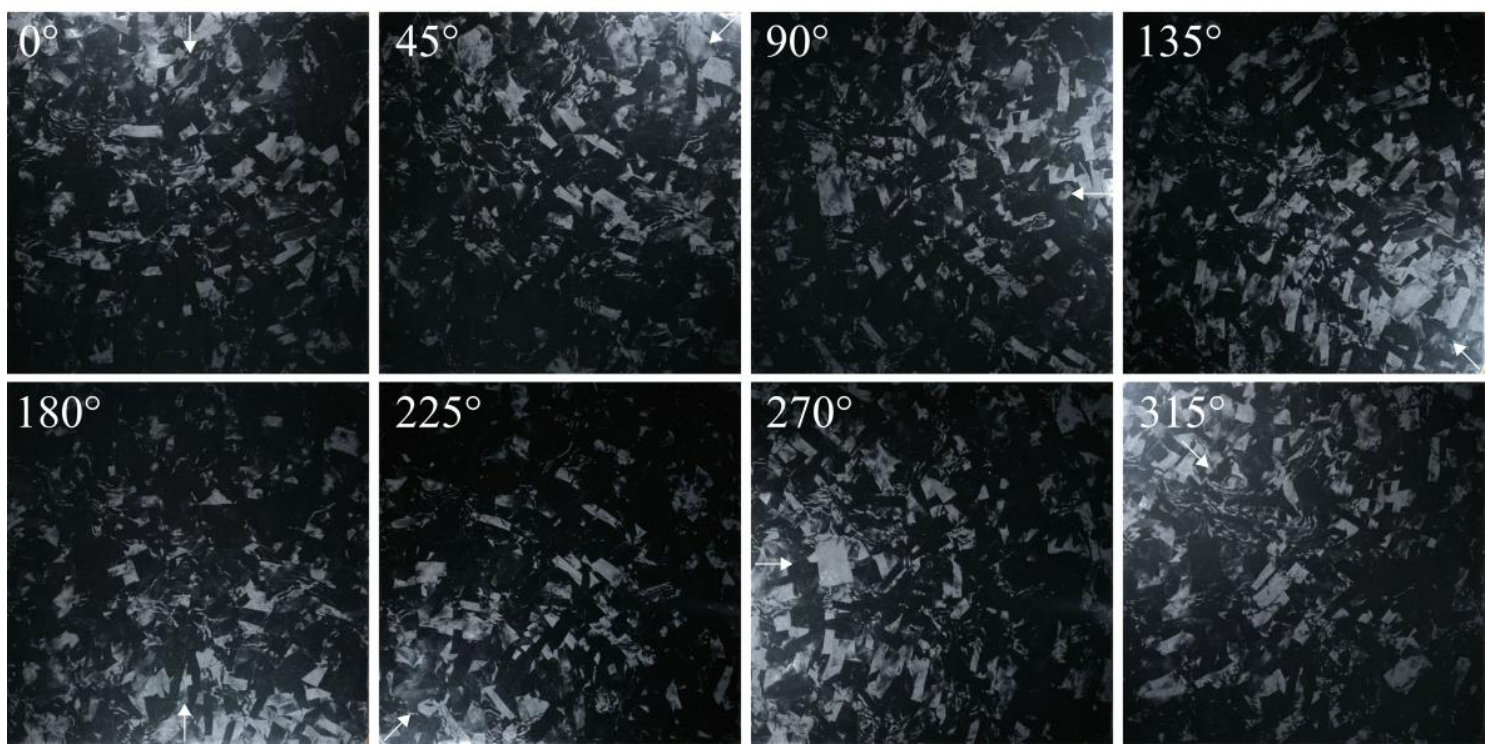

Figure 10: Raw images ( 8 of 32 total) taken with light source positioned at various orientations relative to the discontinuous fibre composite sample (light direction indicated by arrows).

a)

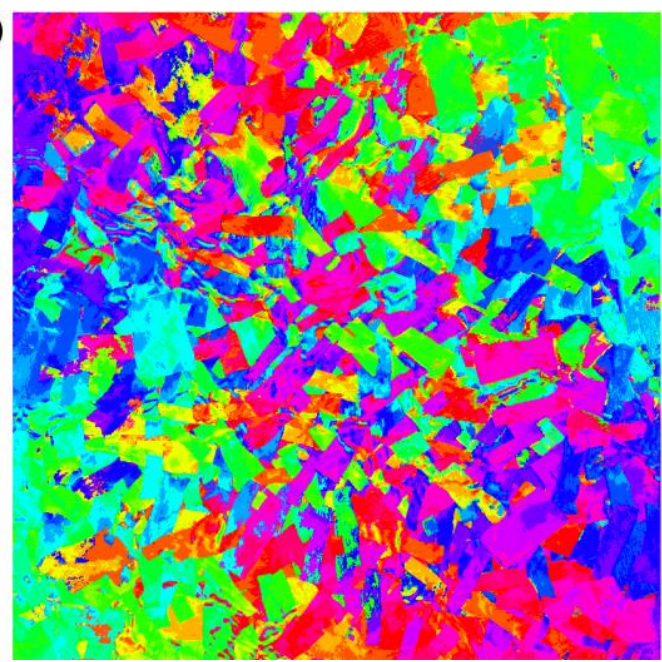

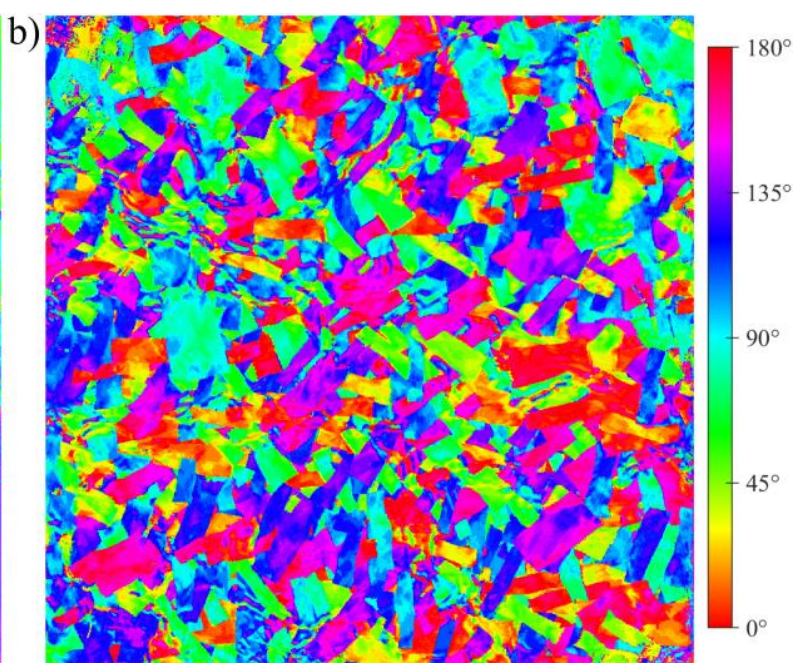

Figure 11: Fibre orientations in the discontinuous fibre composite surface determined by, a) the Maximum Peak Method and, b) the Average Peak Method.

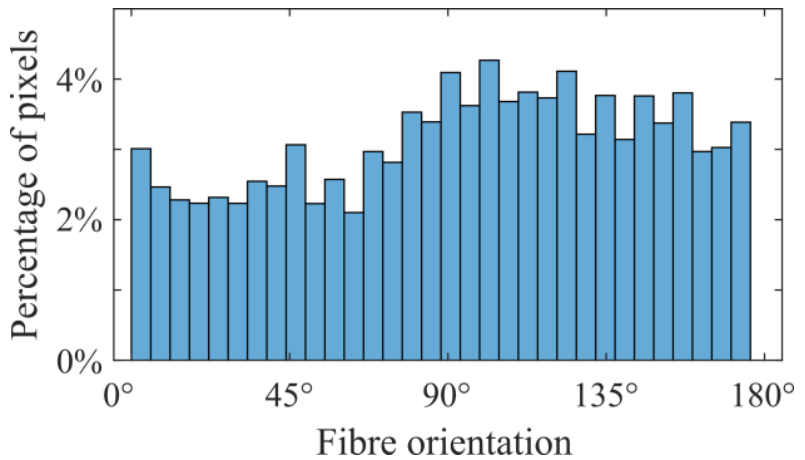


Figure 12: Histogram of fibre orientations in the discontinuous fibre composite surface determined by the Average Peak Method.

a)

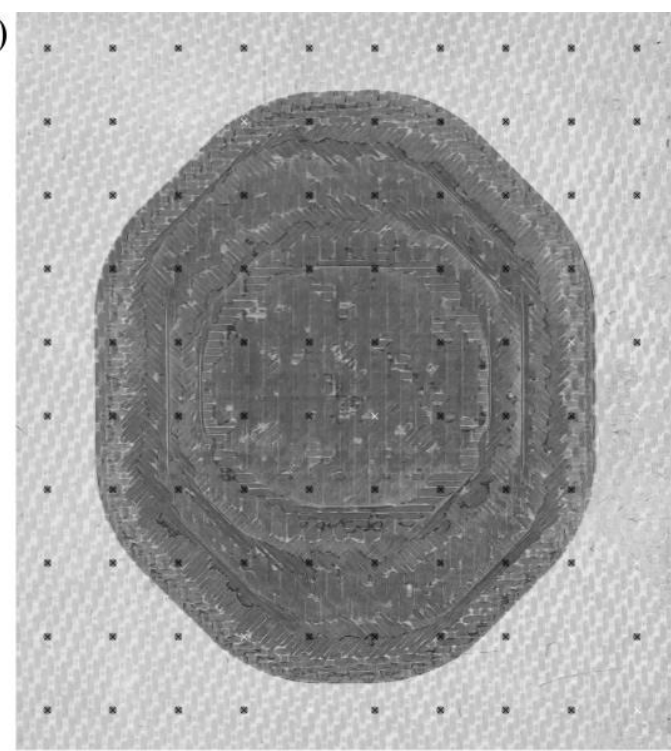

b)

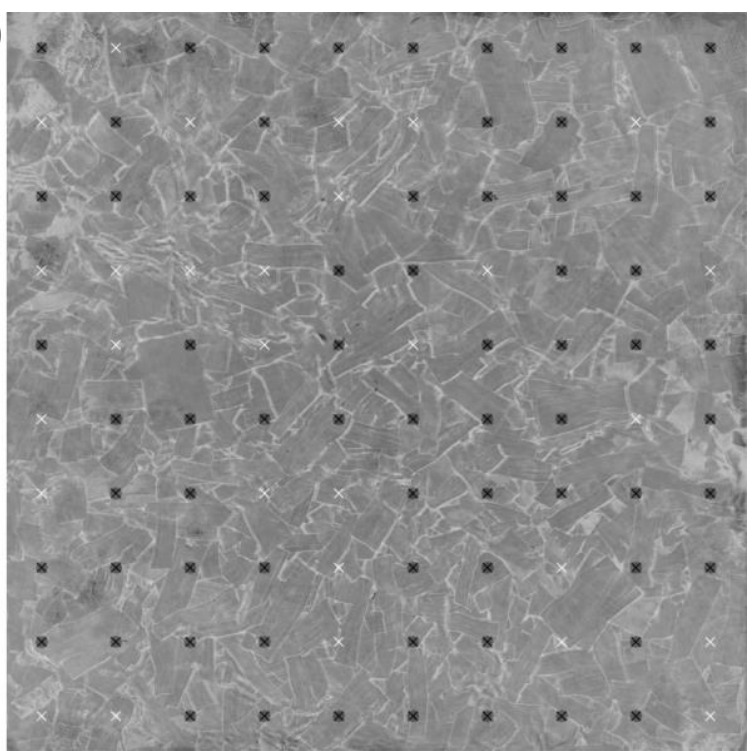

Figure 13: Points for manual fibre orientation measurement from, a) the machined scarf repair surface and, b) the discontinuous fibre composite surface. The black measurement points are shown on an inverse representation of peak reflection intensity (dark areas represent high intensity reflections; light areas represent low intensity reflections).

a)

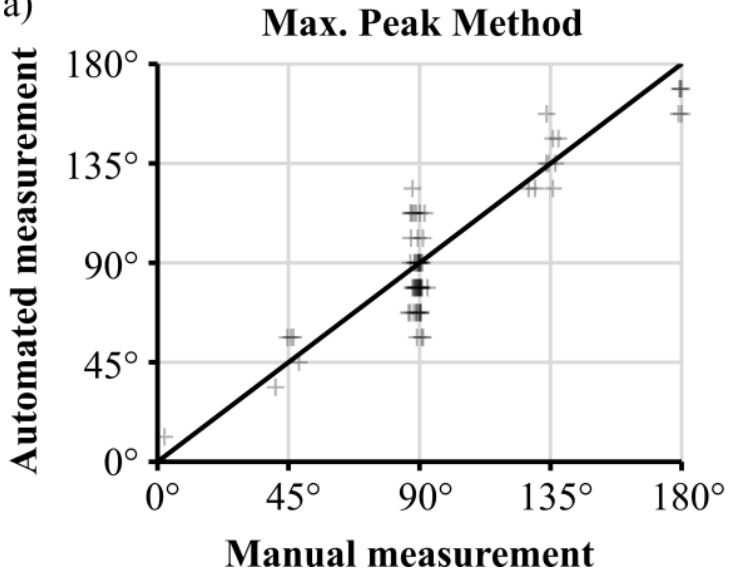

b)

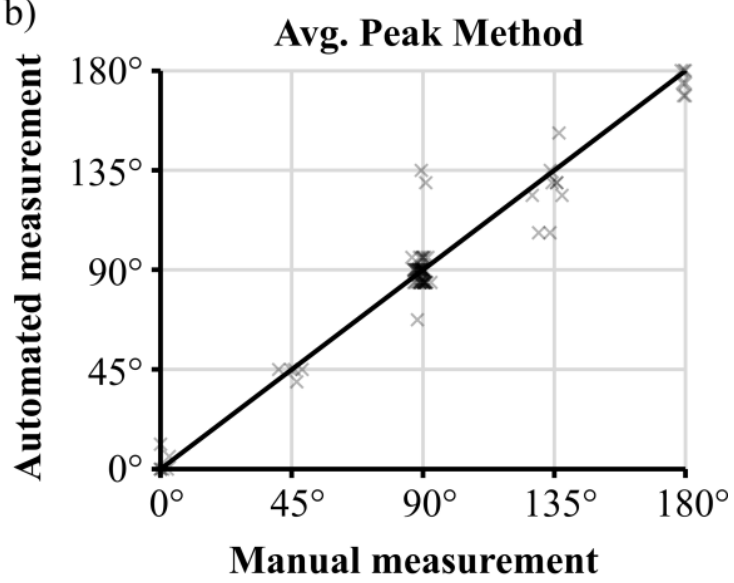

Figure 14: Comparison of calculated and measured fibre orientations in the machined scarf repair surface determined by, a) the Maximum Peak Method and, b) the Average Peak Method based on the use of 32 images. 
a)

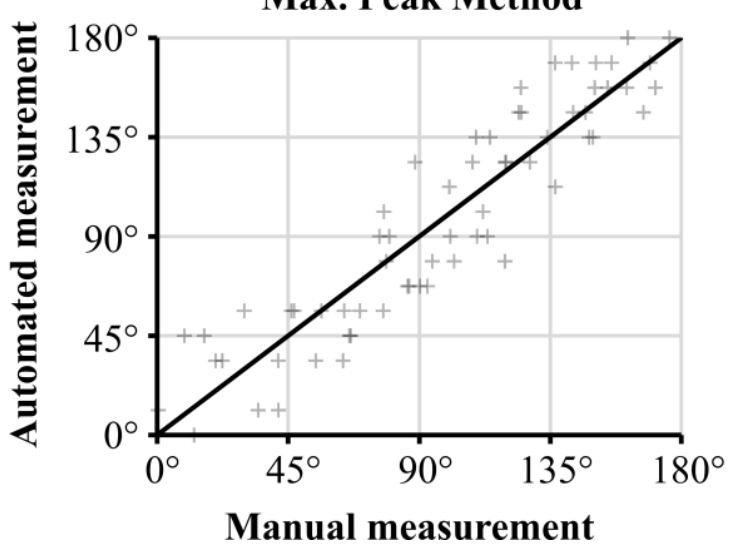

b)



Figure 15: Comparison of calculated and measured fibre orientations in the discontinuous fibre composite surface determined by, a) the Maximum Peak Method and, b) the Average Peak Method based on the use of 32 images.

a)

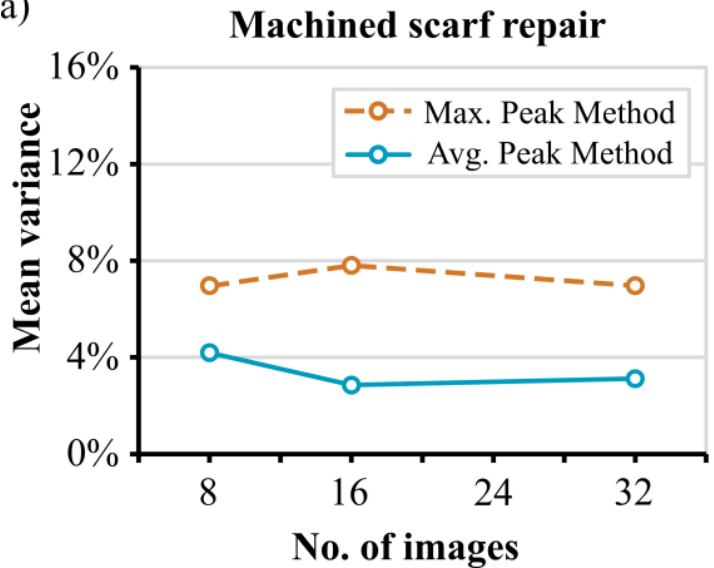

b)

b) Discontinuous fibre composite

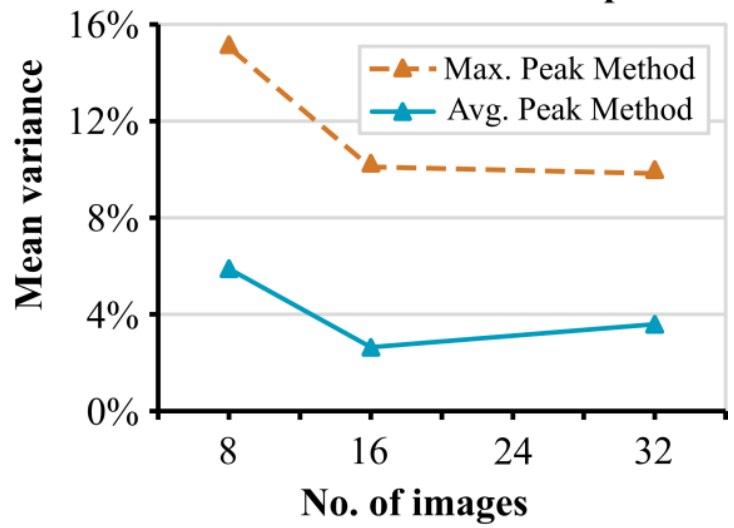

Figure 16: Mean variance between calculated and measured fibre orientations using different methods for, a) the machined scarf repair and, b) the discontinuous fibre composite surfaces, relative to the number of images used in the calculation. 